\title{
Coherent control of atomic tunnelling
}

\author{
John Martin and Daniel Braun \\ Laboratoire de Physique Théorique, IRSAMC, UMR 5152 du CNRS, Université Paul Sabatier, Toulouse, \\ France \\ E-mail: jmartin@irsamc.ups-tlse.fr and braun@irsamc.ups-tlse.fr
}

Received 21 February 2008, in final form 17 April 2008

Published 20 May 2008

Online at stacks.iop.org/JPhysB/41/115502

\begin{abstract}
We study the tunnelling of a two-level atom in a double well potential while the atom is coupled to a single electromagnetic field mode of a cavity. The coupling between internal and external degrees of freedom, due to the mechanical effect on the atom from photon emission into the cavity mode, can dramatically change the tunnelling behaviour. We predict that in general the tunnelling process becomes quasiperiodic. In a certain regime of parameters a collapse and revival of the tunnelling occurs. Accessing the internal degrees of freedom of the atom with a laser allows us to coherently manipulate the atom position, and in particular to prepare the atom in one of the two wells.
\end{abstract}

(Some figures in this article are in colour only in the electronic version)

\section{Introduction}

The tunnelling effect is considered one of the hallmarks of quantum mechanical behaviour. Historically, tunnelling was first examined for single particles (e.g. $\alpha$ particles [1], electrons in field emission [2] and later in mesoscopic circuits [3]), for Cooper pairs [4] and for molecular groups [5-7]. Recently the tunnelling of atoms has attracted substantial attention [8-11]. Dynamical (chaos assisted) tunnelling of ultracold atoms between different islands of stability in phase space was analysed in [12,13] and has been observed experimentally $[14,15]$. The resonantly enhanced tunnelling of atoms between wells of a tilted optical lattice has also been observed very recently [16]. In all of these examples, the atoms have been considered internally as inert, and only the centre-of-mass coordinate of the atom was of interest. In [17] it was shown that by taking into account the internal degrees of freedom of atoms, an atom/optical double well potential could be created in which tunnelling atoms see their internal and external states correlated (such an effect is also known from other contexts [18]). Mechanical effects of light in optical resonators were also investigated in [19], but no tunnelling was considered.

Here we show that the tunnelling effect can be drastically modified if an internal transition of the atom is coupled to a single electromagnetic mode in a cavity, such that photon emission is a reversible and coherent process. The resulting Rabi oscillations between states with the excitation in the atom and states with a photon in the cavity modulate the periodic tunnelling motion. Depending on the frequencies involved, a rich quasi-periodic behaviour can result. If the cavity is fed with a coherent state, collapse and revival of the tunnelling effect can occur. Moreover, we show that one may profit from access to the internal degrees of freedom of the atom (e.g. with a laser) to control the atomic motion in the external potential.

\section{Model}

\subsection{Derivation of the Hamiltonian}

Consider a trapped two-level atom (with levels $|g\rangle,|e\rangle$ of energy $\mp \hbar \omega_{0} / 2$ respectively) interacting with a standing wave (with wave number $k$ and frequency $\omega$ ) inside a cavity as illustrated in figure 1. The atom is assumed to be bound in the $y-z$, plane at the equilibrium position $y=z=0$ and to experience a symmetric double well potential $V(x)$ along the $x$ direction. We denote by $\Delta$ the tunnel splitting, i.e. the energy spacing between the two lowest energy states (the symmetric $|-\rangle$ and antisymmetric $|+\rangle$ states) of this double well potential. Below we also allow the trapped atom to interact resonantly with an external laser. The Hamiltonian of this system is given by

$$
H=H_{\mathrm{A}}+H_{\mathrm{F}}+H_{\mathrm{AF}},
$$

where $H_{\mathrm{A}}=H_{\mathrm{A}}^{\mathrm{ex}}+H_{\mathrm{A}}^{\mathrm{in}}$ is the Hamiltonian of the trapped atom, $H_{\mathrm{F}}$ is the Hamiltonian of the free field and $H_{\mathrm{AF}}$ is the 

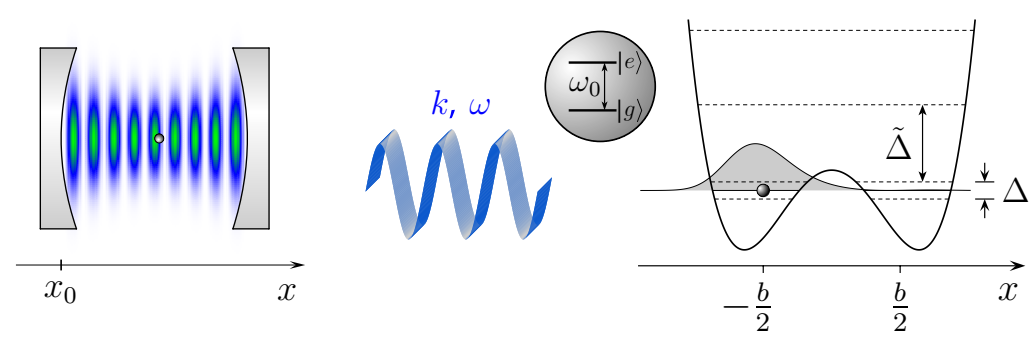

Figure 1. Two-level atom in a double well potential interacting with a standing wave inside a cavity.

interaction Hamiltonian describing the atom-field interaction. We have

$$
\begin{aligned}
H_{\mathrm{A}}^{\mathrm{ex}} & =\frac{p_{x}^{2}}{2 m}+V(x), & H_{\mathrm{A}}^{\mathrm{in}} & =\frac{\hbar \omega_{0}}{2} \sigma_{z}^{\mathrm{in}}, \\
H_{\mathrm{F}} & =\hbar \omega a^{\dagger} a, & H_{\mathrm{AF}} & =-\mathbf{d} \cdot \mathbf{E},
\end{aligned}
$$

where $\mathbf{d}$ denotes the atomic dipole,

$$
\mathbf{E}=E_{\omega} \varepsilon\left(a+a^{\dagger}\right) \sin \left(k\left(x-x_{0}\right)\right)
$$

is the electric field operator, with $E_{\omega}=\sqrt{\frac{\hbar \omega}{\epsilon_{0} V}}$, where $\epsilon_{0}$ is the permittivity of free space, $V$ the electromagnetic mode volume, $x_{0}$ the abscissa at the left cavity mirror $\left(x_{0}<0\right)$, and $\varepsilon$ the electric field polarization vector. We have introduced the operators $\sigma_{i}^{\text {in }}$ (resp. $\sigma_{i}^{\text {ex }}$ ) for $i=x, y, z$ as the Pauli spin operators in the basis $\{|e\rangle,|g\rangle\}$ (resp. $\{|+\rangle,|-\rangle\}$ ). The operator $x$ stands for the centre-of-mass position of the atom, $p_{x}$ is the conjugate momentum along the $x$-axis, $m$ denotes the atomic mass and $a\left(a^{\dagger}\right)$ the annihilation (creation) operator of the cavity radiation field.

We adopt the two-level approximation which consists of taking into account only the two lowest motional energy states. This requires the double-well separation to be much smaller than the wavelength of the cavity mode, and the Rabi frequency $\sqrt{4 g^{2}+\delta^{2}}$ (with $\delta=\omega-\omega_{0}$ the detuning between the cavity field and the atomic transition frequencies) to be much smaller than the frequency gap $\tilde{\Delta}$ between the upper motional states and the ground-state doublet (see figure 1). Within this approximation, Hamiltonian $H_{\mathrm{A}}^{\mathrm{ex}}$ becomes

$$
H_{\mathrm{A}}^{\mathrm{ex}}=\frac{\hbar \Delta}{2} \sigma_{z}^{\mathrm{ex}}
$$

and the position operator takes the form $x=\frac{b}{2} \sigma_{x}^{\text {ex }}$ with $b / 2=$ $\langle+|x|-\rangle$. We can form states that are mainly concentrated in the left/right wells,

$$
|L\rangle=(|+\rangle-|-\rangle) / \sqrt{2}, \quad|R\rangle=(|+\rangle+|-\rangle) / \sqrt{2} .
$$

The average position of a particle localized in the right well is then given by $b / 2$ (see figure 1) and $\sigma_{x}^{\mathrm{ex}}=|R\rangle\langle R|-| L\rangle\langle L|$. The interaction Hamiltonian $H_{\mathrm{AF}}$ can then be written

$$
H_{\mathrm{AF}}=-\hbar g\left(a+a^{\dagger}\right)\left[\sin \chi \cos \kappa \sigma_{x}^{\mathrm{in}}-\cos \chi \sin \kappa \sigma_{x}^{\mathrm{ex}} \sigma_{x}^{\mathrm{in}}\right]
$$

with the atom-field coupling strength $g=-\langle e|\mathbf{d}| g\rangle \cdot \varepsilon E_{\omega} / \hbar$, and

$$
\chi=k x_{0}, \quad \kappa=k b / 2 .
$$

For long wavelengths $(\kappa \ll 1)$, or $\kappa=n \pi$ with integer $n$, the left and right sites of the double well are indistinguishable to the cavity photon and $H_{\mathrm{AF}}$ reduces to JaynesCummings Hamiltonian without rotating wave approximation (with a sine varying coupling constant), $-\hbar g \sin \chi\left(a+a^{\dagger}\right) \sigma_{x}^{\text {in }}$. Note that $\kappa \ll 1$ would normally be identified with the LambDicke regime. Here the situation is more subtle as the level spacings between the tunnelling split ground-state doublet and the next excited states can be very different such that the recoil energy $\hbar \omega_{\text {recoil }}$ satisfies $\Delta \ll \omega_{\text {recoil }} \ll \tilde{\Delta}$. One may thus be in the Lamb-Dicke regime concerning transitions to higher vibrational states but have a significant mechanical effect on the atomic tunnelling. Furthermore, since there is only one photon mode, the recoil energy cannot vary continuously and exciting higher vibrational levels requires $\omega_{\text {recoil }}$ close to a level spacing. Our numerical calculations show that even for $\kappa \sim 1$ the two-level approximation can still work very well (see figure 4).

For $\delta, \Delta \ll \omega, \omega_{0}$, a rotating wave approximation is justified, which consists in eliminating the energy non-conserving terms $a \sigma_{ \pm}^{\text {ex }} \sigma_{-}^{\text {in }}$ and $a^{\dagger} \sigma_{ \pm}^{\text {ex }} \sigma_{+}^{\text {in }}$ with $\sigma_{+}^{\text {in }}=$ $|e\rangle\langle g|, \sigma_{-}^{\mathrm{in}}=\sigma_{+}^{\mathrm{in} \dagger}$ and $\sigma_{+}^{\mathrm{ex}}=|+\rangle\langle-|, \sigma_{-}^{\mathrm{ex}}=\sigma_{+}^{\mathrm{ex} \dagger}$. Within this approximation, the total Hamiltonian reads

$$
\begin{aligned}
H= & \frac{\hbar \Delta}{2} \sigma_{z}^{\mathrm{ex}}+\frac{\hbar \omega_{0}}{2} \sigma_{z}^{\mathrm{in}}+\hbar \omega a^{\dagger} a+\hbar g\left(a \sigma_{+}^{\mathrm{in}}+a^{\dagger} \sigma_{-}^{\mathrm{in}}\right) \\
& \times\left[\cos \chi \sin \kappa \sigma_{x}^{\mathrm{ex}}-\sin \chi \cos \kappa 1^{\mathrm{ex}}\right]
\end{aligned}
$$

Thus, depending on the parameters $\chi$ and $\kappa$, the cavity photon may induce internal transitions in the atom only $(\cos \chi \sin \kappa=0)$, or induce transitions between internal and external states at the same time $(\cos \chi \sin \kappa \neq 0)$ even for a vanishing detuning $\left(\delta=\omega-\omega_{0}=0\right)$. This is in contrast to conventional sideband transitions of harmonically bound atoms or ions in the Lamb-Dicke regime which require an appropriate value of the detuning. For a fixed potential centre (and thus fixed $\chi$ ), $\kappa$ can be changed through a modulation of the well-to-well separation $b$. We will neglect in the following the effects of decoherence, which means that not only $g$ but also $\Delta$ should be much larger than the rate of spontaneous emission $\Gamma$, and the cavity decay rate $\kappa_{\text {cav }}$. The effects of the decoherence of an atom tunnelling in a double well potential but without cavity and thus coupling to the full 3D continuum of electromagnetic modes was studied in [20].

We denote the global state of the atom-field system by $|n, i, j\rangle \equiv|n\rangle \otimes|i\rangle \otimes|j\rangle$ where $|n\rangle$ stands for the cavity field eigenstates, $|i\rangle \in\{|-\rangle,|+\rangle\}$ for the external motional states, 
and $|j\rangle \in\{|g\rangle,|e\rangle\}$ for the internal states. The total excitation number $N$ is given by $a^{\dagger} a+\sigma_{+}^{\text {in }} \sigma_{-}^{\text {in }}$.

\subsection{Energy levels}

The states $|0, \pm, g\rangle$ are eigenstates of $H$ with eigenvalue $\left(-\hbar \omega_{0} \pm \hbar \Delta\right) / 2$, i.e. these states remain uncoupled and represent the two lowest energy states in the regime $\delta$, $\Delta \ll \omega, \omega_{0}$. It is straightforward to verify that the Hamiltonian (9) only induces transitions between states with the same number of excitations $N,\{|N-1,+, e\rangle,|N,+, g\rangle, \mid N-$ $1,-, e\rangle,|N,-, g\rangle\} \equiv\{|1\rangle,|2\rangle,|3\rangle,|4\rangle\} . \quad$ It is therefore sufficient to solve the dynamics in this subspace. In doing so, we obtain the eigenvalues of $H$,

$$
\lambda_{\rho \mu}=(N-1 / 2) \hbar \omega+\rho \frac{\hbar \Omega_{\mu}}{2},
$$

for $\rho, \mu \in\{ \pm\}, N=1,2, \ldots$, and with

$\Omega_{ \pm}=\sqrt{2 N g^{2}(1-\cos (2 \kappa) \cos (2 \chi))+\delta^{2}+\Delta^{2} \pm 2 \Omega^{2}}$,

$\Omega^{2}=\sqrt{4 N g^{2} \cos ^{2} \kappa \sin ^{2} \chi\left(\Delta^{2}+4 N g^{2} \sin ^{2} \kappa \cos ^{2} \chi\right)+\delta^{2} \Delta^{2}}$.

For a vanishing tunnel splitting $(\Delta=0), \Omega_{ \pm}$reduces to the maximum (minimum) of the two Rabi frequencies of the Jaynes-Cummings models in the right and left wells. For $\cos \kappa=1$, the decoupling of external and internal degrees of freedom manifests itself also in the eigenvalues with $\Omega_{ \pm}=\left|\sqrt{4 N g^{2} \sin ^{2} \chi+\delta^{2}} \pm \Delta\right|$.

\subsection{Evolution operator}

The whole dynamics of the system can be described by means of the evolution operator $U(t)=\mathrm{e}^{-\mathrm{i} H t / \hbar}$ with components $U_{i j}=\langle i|U(t)| j\rangle=U_{j i}$, which can be calculated exactly. In order to simplify the expressions, we restrict ourselves in the following to $\chi=-\pi / 4-2 n \pi$ (integer $n$ ). We find, up to a an overall phase $\mathrm{e}^{-\mathrm{i}(N-1 / 2) \omega t}$,

$$
\begin{aligned}
U_{11} & =-\frac{\mathrm{i}}{2 \Lambda} \sum_{\mu= \pm}\left[\mu S_{\mu} \Omega_{-\mu}\left\{\xi+\mu(\Delta-\delta) \Omega^{2}\right\}\right. \\
& \left.-\mathrm{i} \mu \Omega_{+} \Omega_{-} C_{\mu}\left(\delta \Delta-\mu \Omega^{2}\right)\right] \\
U_{12} & =\frac{-\mathrm{i} \sqrt{N} g \cos \kappa}{\sqrt{2} \Lambda} \sum_{\mu= \pm}\left[\mu S_{\mu} \Omega_{-\mu}\right. \\
& \left.\times\left(\Delta^{2}+2 N g^{2} \sin ^{2} \kappa+\mu \Omega^{2}\right)+\mathrm{i} \mu \Omega_{+} \Omega_{-} \Delta C_{\mu}\right] \\
U_{13} & =\frac{-\mathrm{i} N g^{2} \sin (2 \kappa)}{2 \Lambda} \sum_{\mu= \pm}\left[\mu \delta \Omega_{\mu} S_{-\mu}+\mathrm{i} \mu \Omega_{+} \Omega_{-} C_{\mu}\right] \\
U_{23} & =\frac{\mathrm{i} \sqrt{N} g \sin \kappa}{\sqrt{2} \Lambda} \sum_{\mu= \pm}\left[\mu \Omega_{\mu} S_{-\mu}\left(\delta \Delta+2 N g^{2} \cos ^{2} \kappa-\mu \Omega^{2}\right)\right]
\end{aligned}
$$

with

$$
\xi=\Delta\left(\delta^{2}+2 N g^{2} \cos ^{2} \kappa-\delta \Delta\right), \quad \Lambda=\Omega_{+} \Omega_{-} \Omega^{2},
$$

and where all time dependence is in the coefficients

$$
C_{ \pm}=\cos \left(\Omega_{ \pm} t / 2\right), S_{ \pm}=\sin \left(\Omega_{ \pm} t / 2\right) .
$$

The remaining components can be deduced from the relations $U_{22}(\delta, \Delta)=U_{33}(-\delta,-\Delta)=U_{44}(\delta,-\Delta)=U_{11}(-\delta, \Delta)$, $U_{24}(\delta, \Delta)=U_{13}(-\delta, \Delta), U_{14}(\delta, \Delta)=U_{23}(\delta,-\Delta)=$ $U_{23}(-\delta, \Delta)$ and $U_{34}(\delta, \Delta)=U_{12}(\delta,-\Delta)$, valid for any $\chi$, where we have made explicit the dependence of $U_{i j}$ on $\delta$ and $\Delta$.

\section{Internal and external dynamics}

The reduced density matrix $\rho^{\text {ex }}$ for the atomic centre-of-mass motion alone follows from $\rho=|\psi(t)\rangle\langle\psi(t)|$ by tracing out the field and internal degrees of freedom, where the total wavefunction at time $t$ reads $|\psi(t)\rangle=\sum_{i, j=1}^{4} U_{i j}\langle j \mid \psi(0)\rangle|i\rangle$. The average position of the atom in the double well potential is then given by

$$
\langle x\rangle=\frac{b}{2} \operatorname{Tr}_{\mathrm{ex}}\left(\rho^{\mathrm{ex}} \sigma_{x}^{\mathrm{ex}}\right)=\frac{b}{2}\left(1-2 \rho_{L L}\right)
$$

with $\rho_{L L}=\left\langle L\left|\rho^{\mathrm{ex}}\right| L\right\rangle$. Similarly, we obtain the reduced density matrix $\rho^{\text {in }}$ for the internal atomic state by tracing out the field and external degrees of freedom, and the probability to find the atom in the excited state as $\rho_{e e}=\left\langle e\left|\rho^{\text {in }}\right| e\right\rangle$.

In the following, we first focus on resonant atom-field interaction $\left(\omega=\omega_{0}\right)$ before moving to the non-resonant case $\left(\omega \neq \omega_{0}\right)$. We distinguish three regimes according to the tunnel splitting compared to the Rabi frequency $g$ : the small tunnel splitting regime (when $\Delta / g \ll 1$ ), the intermediate regime (when $\Delta / g \sim 1$ ), and the large tunnel splitting regime (when $\Delta / g \gg 1$ ).

\subsection{Resonant atom-field interaction}

For the resonant atom-field interaction $(\delta=0)$, the expressions for $U_{i j}$ can be greatly simplified. If the system is initially prepared in the state $|N-1, R, e\rangle$ and for $\kappa=\pi / 4$, we have

$$
\rho_{L L}=\frac{\Delta^{2}}{\Delta^{2}+N g^{2}} \sin ^{2}\left(\frac{\Omega_{\mathrm{tun}} t}{2}\right)
$$

with the tunnel frequency

$$
\Omega_{\mathrm{tun}}=\frac{1}{2}\left(\Omega_{+}+\Omega_{-}\right)
$$

and

$\rho_{e e}=\frac{1}{2}+\frac{\sum_{\mu= \pm}\left(\Omega_{\mu}^{2}-\Delta^{2}\right) \cos \left(\Omega_{\mu} t\right)+4 \Delta^{2} \cos \left(\frac{\Omega_{+}-\Omega_{-}}{2} t\right)}{8\left(N g^{2}+\Delta^{2}\right)}$.

The atom position oscillates with a single frequency $\Omega_{\text {tun }}$ given by equation (18), whereas $\rho_{e e}$ evolves with three in general incommensurable frequencies $\Omega_{+}, \Omega_{-}$and $\left(\Omega_{+}-\Omega_{-}\right) / 2$ giving rise to a quasi-periodic signal.

For $\Delta / g \ll 1$, equation (17) leads to $\rho_{L L} \simeq 0$ (up to order $\left.(\Delta / g)^{2}\right)$, indicating that tunnelling is suppressed. This is already obvious from (9), as the term responsible for tunnelling, $(\hbar \Delta / 2) \sigma_{z}^{\mathrm{ex}}=(\hbar \Delta / 2)(|R\rangle\langle L|+| L\rangle\langle R|)$ becomes very small compared to the last term, diagonal in $|R\rangle,|L\rangle$ which leads to internal Rabi flopping. Note, however, that tunnelling is suppressed on all time scales, even for $t \gg 1 / \Delta$, due to the reduced amplitude in equation (17), very much 


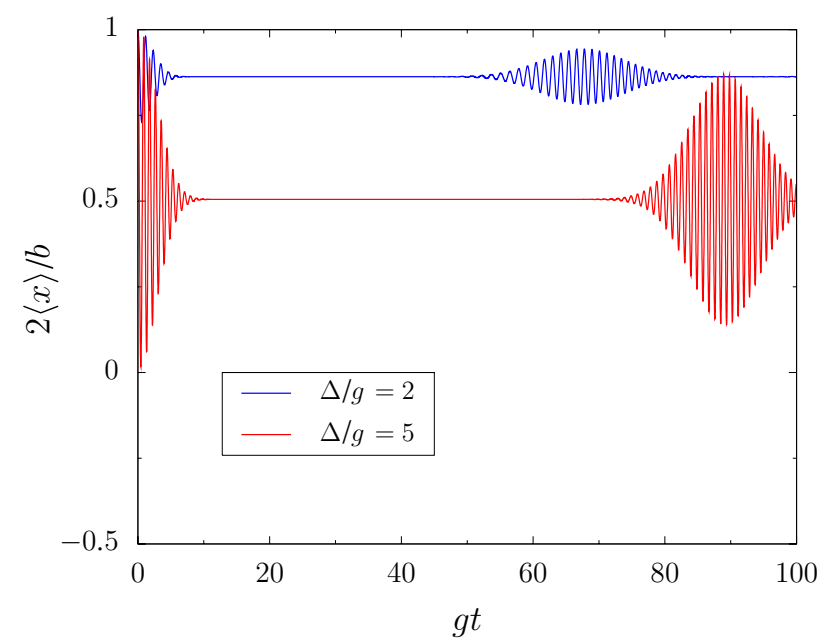

Figure 2. Average position of the atom in the double well as a function of time for $\Delta / g=2$ (blue, top curve) and $\Delta / g=5$ (red), $\kappa=\pi / 4$ and a coherent state with $\alpha=5$.

in contrast to tunnelling without internal degrees of freedom, where only the period of the tunnelling motion, but not the amplitude is affected when $\Delta$ is reduced. For $\kappa$ approaching $\pi$, the situation changes because the term $g \cos \chi \sin \kappa \sigma_{x}^{\text {ex }}$ of the interaction Hamiltonian inducing transitions between vibrational states becomes small in comparison with $\Delta$ thereby allowing tunnelling again.

Because internal and external degrees of freedom are coupled, the tunnelling frequency (equation (18)) depends on the number of photons inside the cavity. As an example, let us now consider $\Delta \sim g$ and a cavity field initially in a coherent state $|\alpha\rangle=\mathrm{e}^{-\frac{1}{2}|\alpha|^{2}} \sum_{n=0}^{\infty} \frac{\alpha^{n}}{\sqrt{n !}}|n\rangle$ with $|\alpha|^{2}$ equal to the mean photon number $\langle n\rangle$. Figure 2 shows that the average position of the atom in the double well as a function of time for a coherent state exhibits collapses and revivals. The oscillation amplitude decreases with increasing mean photon number $\langle n\rangle$ and decreasing tunnel splitting $\Delta$ (see equations (11)-(17)). Since the probability to find the atom in the excited state oscillates with three frequencies, no collapses and revivals are observed for $\rho_{e e}$.

The collapse time $t_{c}$ of the tunnelling motion can be estimated from the condition [21] $\left(\Omega_{\mathrm{tun}}(\langle n\rangle+\sqrt{\langle n\rangle})-\right.$ $\left.\Omega_{\text {tun }}(\langle n\rangle-\sqrt{\langle n\rangle})\right) t_{c} \sim 1$ with $\Omega_{\text {tun }}(m)$ given by equation (18) for $N=m+1$, which yields, for $\langle n\rangle \gg 1$,

$$
t_{c} \sim \frac{1}{g}\left(1+\frac{(\Delta / g)^{2}+3 / 4}{2\langle n\rangle}\right)+\mathcal{O}\left(\langle n\rangle^{-2}\right) \text {. }
$$

The time interval between the two following revivals, $t_{r}$, follows from

$$
\left(\Omega_{\mathrm{tun}}(\langle n\rangle)-\Omega_{\mathrm{tun}}(\langle n\rangle-1)\right) t_{r}=2 \pi,
$$

and is given for $\langle n\rangle \gg 1$ by

$$
t_{r} \simeq \frac{4 \pi \sqrt{\langle n\rangle}}{g}\left(1+\frac{(\Delta / g)^{2}+1 / 2}{2\langle n\rangle}+\mathcal{O}\left(\langle n\rangle^{-2}\right)\right) .
$$

For the parameters of figure 2, equation (22) yields $g t_{r} \simeq 68.23$ for $\Delta / g=2$ and $g t_{r} \simeq 86.70$ for $\Delta / g=5$. Smaller revival times are possible for smaller values of $\langle n\rangle$, but in general

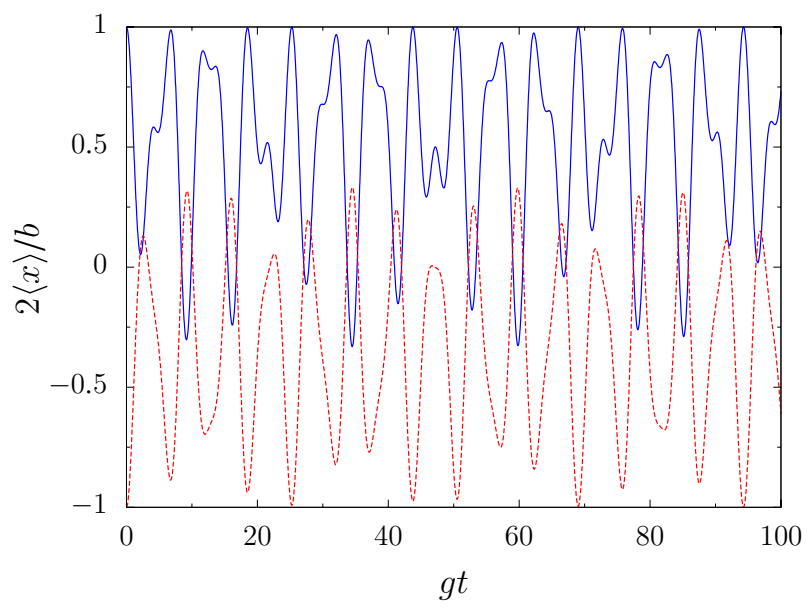

Figure 3. Average position of the atom in the double well as a function of time for $\Delta=\delta=g, \kappa=\pi / 4$ and $N=1$. The blue solid/red dashed curve corresponds to an excited atom initially located in the right/left well.

the observation of revivals will be quite challenging, as they require $\Delta \sim g \gg \kappa_{\text {cav }}$ (see section 4 ).

For large tunnel splitting, $\Delta / g \gg 1, \Omega_{\text {tun }}=\Delta+$ $N g^{2} /(2 \Delta)+\mathcal{O}\left((g / \Delta)^{3}\right)$, and equation (17) reduces to $\rho_{L L} \simeq$ $\sin ^{2}(\Delta t / 2)$, which is identical to the tunnelling of a particle without internal structure. Equation (19) reduces to a Rabi oscillation $\rho_{e e} \simeq \cos ^{2}(\sqrt{N} g t / 2)$.

\subsection{Non-resonant atom-field interaction}

For non-resonant atom-field interaction $(\delta \neq 0)$, and intermediate tunnel splitting (see figure 3 for $\Delta=\delta=$ $g),\langle x(t)\rangle$ involves in general the two non-commensurate frequencies $\Omega_{+}$and $\Omega_{-}$and varies therefore quasiperiodically as a function of time. Figure 3 also shows that an atom initially located in one of the two wells remains mostly confined to that well.

For small tunnel splitting, $\Delta / g \ll 1$ and large detuning $|\delta| / g \gg 1$ (with $\Delta|\delta| / g^{2} \sim 1$ ), the matrix elements of $U$ simplify to

$$
\begin{aligned}
& U_{13}=\frac{\mathrm{i} N g^{2} \sin 2 \kappa}{\sqrt{\delta^{2} \Delta^{2}+N^{2} g^{4} \sin ^{2}(2 \kappa)}} \sin \left(\frac{\bar{\Omega} t}{2}\right) \\
& U_{33}=\cos \left(\frac{\bar{\Omega} t}{2}\right)+\frac{\mathrm{i} \delta \Delta}{\sqrt{\delta^{2} \Delta^{2}+N^{2} g^{4} \sin ^{2}(2 \kappa)}} \sin \left(\frac{\bar{\Omega} t}{2}\right)
\end{aligned}
$$

up to corrections of order $\mathcal{O}(\Delta / g)$ and a phase factor $\mathrm{e}^{\mathrm{i}\left[\left(N g^{2} / \delta+\delta\right)-(2 N-1) \omega\right] t / 2}$ while the components $U_{12}$ and $U_{23}$ are of order $\mathcal{O}(\Delta / g)$. In this situation, the system oscillates only between the two states $|N-1,+, e\rangle$ and $|N-1,-, e\rangle$ with a single frequency

$$
\bar{\Omega}=\frac{\sqrt{\delta^{2} \Delta^{2}+N^{2} g^{4} \sin ^{2}(2 \kappa)}}{\delta},
$$

just as a three-level atom undergoing a Raman transition in the far detuned regime behaves as a two-level system. 
If the system is initially in the state $|N-1,-, e\rangle$, we have from equations (23) and (24)

$$
\rho_{L L}=\frac{1}{2}-\frac{N \delta \Delta \sin (2 \kappa)}{2 \bar{\Omega}^{2}(\delta / g)^{2}}[1-\cos (\bar{\Omega} t)]
$$

and $\rho_{e e}=1$. For a detuning $\delta= \pm N g^{2} \sin (2 \kappa) / \Delta$,

$$
\rho_{L L}=\frac{1}{2} \mp \frac{1}{4}[1-\cos (\sqrt{2} \Delta t)] .
$$

This regime may be suitable for coherently manipulating the atom position through access to its internal degrees of freedom with a laser. Coherent manipulation of the position of neutral atoms has been proposed and demonstrated before, see, e.g. [22, 24]. In these examples, the manipulation is done by modifying the external potential. The mechanism we propose here is very different, as the potential remains totally unchanged, and only internal transitions and the tunnelling effect are used to move the atom in a controlled way. As an example, we show how the atom can be prepared in the left well starting from the ground state $|0,-, g\rangle$ for $\delta=-g^{2} / \Delta$.

We first apply a $\pi$-pulse with an external laser resonant with the atomic transition. By using a laser with a wave vector perpendicular to the $O x$-direction, only the atomic internal degree of freedom is affected, resulting in the transition $|0,-, g\rangle \rightarrow-\mathrm{i}|0,-, e\rangle$. We assumed that the laser Rabi frequency $\Omega_{R}$ is much larger than the tunnel frequency $\Delta$.

Now we use the coupling between the internal and external degrees of freedom to create a superposition of the $|0, \pm, e\rangle$ states, and then apply a second resonant $\pi$-pulse to get back to the uncoupled states $|0, \pm, g\rangle$. For $\Delta / g \ll 1, \delta=-g^{2} / \Delta$ and $\kappa=\pi / 4$, the initial state transforms according to

$$
|0,-, g\rangle \stackrel{\Omega_{R} t=\pi}{\longrightarrow}|0,-, e\rangle \stackrel{\Delta t=\pi / \sqrt{2}}{\longrightarrow}|0, L, e\rangle \stackrel{\Omega_{R} t=\pi}{\longrightarrow}|0, L, g\rangle
$$

up to a physically irrelevant phase. Other coherent superpositions of $|0,+, g\rangle$ and $|0,-, g\rangle$ can be obtained by choosing appropriate interaction times.

In order to verify that the two-level approximation for the external motion used in the derivation of the Hamiltonian is a good approximation, we have numerically solved the time-dependent Schrödinger equation with Hamiltonian (1) and rotating wave approximation but with the exact external potential $V(x)$ (i.e. with a large number of vibrational states). Figure 4 shows that provided $\tilde{\Delta} \gg \sqrt{4 g^{2}+\delta^{2}}$ as stated before, to take only the two lowest vibrational states into account is indeed a good approximation.

\section{Possible experimental realization}

The experimental realization of our model is certainly challenging. For completeness of the theoretical investigation, we have explored all possible regimes of the model for a large variety of parameters. The regime that seems most accessible with current technology is the regime of small tunnel splitting $\Delta \ll g$, which is the one most interesting for coherent control of the atom position, but which requires a very high quality cavity and slow spontaneous emission (as has been demonstrated for microwave cavities [25]). Double well potentials with tunable well-to-well separation have been

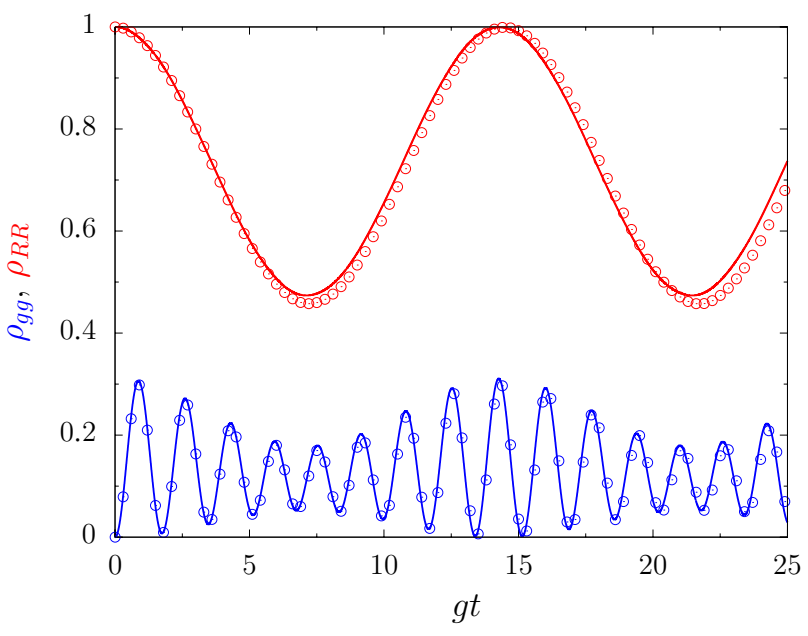

Figure 4. Density matrix elements $\rho_{R R}$ (top) and $\rho_{g g}$ (bottom) as a function of the interaction time $g t$ for an initially excited atom located in the right well and for the parameters $\Delta / g \simeq 0.3336$, $\delta / g=3, \kappa=\pi / 4$ and $N=1$. Numerical results from the propagation of the time-dependent Schrödinger equation with Hamiltonian (1) and rotating wave approximation are represented by circles and analytical results by solid curves. The time propagation was done with $(\hbar=m=1) g=0.01$ and the double well potential $V(x)=0.08 x^{4}-x^{2}$ yielding a tunnel splitting $\Delta \simeq 0.003336$ and a ratio $\tilde{\Delta} / \sqrt{4 g^{2}+\delta^{2}} \simeq 44.4 \gg 1$.

demonstrated with optical dipole traps, e.g. in [26, 22], and on atom chips, e.g. in [27, 28]. For our model, the double well potential has to be realized inside the cavity. Optical trapping and even cooling of atoms close to their ground state inside a cavity has been achieved in several groups [29-32], but up to our knowledge double well potentials have not been realized in a cavity so far. Some of the cavities developed have a very long lateral opening (up to $222 \mu \mathrm{m}$ [33]) and should allow more complicated trapping potentials (optical lattices intersecting a cavity have been realized in Chapman's group [33]). We remark that it is not essential for our model that the double well potential be aligned with the cavity axes. Any other orientation is possible, and only leads to modified coefficients $\cos \chi \sin \kappa$ and $\sin \chi \cos \kappa$.

At certain 'magical wavelengths', $\mathrm{Cs}, \mathrm{Yb}, \mathrm{Sr}, \mathrm{Mg}$ and $\mathrm{Ca}$ atoms in optical traps experience the same potential for ground and excited internal states coupled by a dipole transition [29, 34-36]. In [29] a trap depth $V_{0} / \hbar=47 \mathrm{MHz}$ was achieved inside an optical cavity with $1.2 \mathrm{~mW}$ laser power. Typical tunnelling frequencies in such a potential are of the order of $\mathrm{kHz}$, which is far from the values $g_{0} / 2 \pi=24 \mathrm{MHz}$, $\kappa_{\text {cav }} / 2 \pi=4.2 \mathrm{MHz}$, and $\gamma / 2 \pi=2.6 \mathrm{MHz}$ reported in the same paper. Therefore both $\kappa_{\text {cav }} / \Delta$ and $\gamma / \Delta$ will have to be reduced by three orders of magnitude, before one reaches the regime where decoherence during the tunnelling starts to become negligible. Note, however, that the trap frequency and thus the tunnelling splitting are determined by the laser power and the focusing (or the wavelength for optical lattices), and can therefore be controlled independently of $\Gamma, \kappa_{\mathrm{cav}}$, such that in principle there should be no fundamental problem achieving this regime. 
Other possibilities which might be worthwhile considering are ion traps (where the trapping potential can be tailored independently of the internal degrees of freedom), microwave cavities, or effective two-state systems resulting from Raman transitions. The detection of the tunnelling motion should be possible by optical imaging, i.e. diffusion of laser light from another transition in the optical regime with smaller wavelength than the well separation. Alternatively, one might monitor the transmission through the cavity in the case that it differs for the two locations of the wells [37]. Another possibility might be using the atomic spin as a position meter [17].

\section{Acknowledgments}

We thank Jacques Vigué for an interesting discussion and CALMIP (Toulouse) for the use of their computers. This work was supported by the Agence National de la Recherche (ANR), project INFOSYSQQ, contract number ANR-05-JCJC-0072, and the EC IST-FET project EUROSQIP.

\section{References}

[1] Gamow G 1928 Z. Phys. 51204

[2] Guth E and Mullin C J 1942 Phys. Rev. 61339

[3] Devoret M H, Esteve D, Grabert H, Ingold G-L, Pothier H and Urbina C 1990 Phys. Rev. Lett. 641824

[4] Josephson B D 1974 Rev. Mod. Phys. 46251

[5] Hueller A 1980 Z. Phys. B 36215

[6] Würger A 1989 Z. Phys. B 7665

[7] Braun D and Weiss U 1994 Physica B 202264

[8] Louis A A and Sethna J P 1995 Phys. Rev. Lett. 741363

[9] Meier F and Zwerger W 2001 Phys. Rev. A 64033610

[10] Luxat D L and Griffin A 2002 Phys. Rev. A 65043618

[11] Albiez M, Gati R, Folling J, Hunsmann S, Cristiani M and Oberthaler M K 2005 Phys. Rev. Lett. 95010402

[12] Grossmann F, Dittrich T, Jung P and Hanggi P 1991 Phys. Rev. Lett. 67516

[13] Averbukh V, Osovski S and Moiseyev N 2002 Phys. Rev. Lett. 89253201

[14] Steck D A, Oskay W H and Raizen M G 2001 Science 293274
[15] Hensinger W K et al 2001 Nature 41252

[16] Sias C, Zenesini A, Lignier H, Wimberger S, Ciampini D, Morsch O and Arimondo E 2007 Phys. Rev. Lett. 98120403

[17] Haycock D L, Alsing P M, Deutsch I H, Grondalski J and Jessen P S 2000 Phys. Rev. Lett. 853365

[18] Salzburger T and Ritsch H 2004 Phys. Rev. Lett. 93063002

[19] Domokos P and Ritsch H 2003 J. Opt. Soc. Am. B 201098

[20] Braun D and Martin J 2008 Phys. Rev. A 77032102

[21] Scully M and Zubairy M 1997 Quantum Optics (Cambridge: Cambridge University Press)

[22] Sebby-Strabley J, Anderlini M, Jessen P S and Porto J V 2006 Phys. Rev. A 73033605

[23] Mandel O, Greiner M, Widera A, Rom T, Hänsch T W and Bloch I 2003 Phys. Rev. Lett. 91010407

[24] Mompart J, Eckert K, Ertmer W, Birkl G and Lewenstein M 2003 Phys. Rev. Lett. 90147901

[25] Guerlin C, Bernu J, Deléglise S, Sayrin C, Gleyzes S, Kuhr S, Brune M, Raimond J-M and Haroche S 2007 Nature 448889

[26] Shin Y, Saba M, Pasquini T A, Ketterle W, Pritchard D E and Leanhardt A E 2004 Phys. Rev. Lett. 92050405

[27] Hinds E A, Vale C J and Boshier M G 2001 Phys. Rev. Lett. 861462

[28] Hänsel W, Reichel J, Hommelhoff P and Hänsch T W 2001 Phys. Rev. A 64063607

[29] McKeever J, Buck J R, Boozer A D, Kuzmich A, Nägerl H-C, Stamper-Kurn D M and Kimble H J 2003 Phys. Rev. Lett. 90133602

[30] Ye J, Vernooy D W and Kimble H J 1999 Phys. Rev. Lett. 834987

[31] Sauer J A, Fortier K M, Chang M S, Hamley C D and Chapman M S 2004 Phys. Rev. A 69051804

[32] Maunz P, Puppe T, Schuster I, Syassen N, Pinske P W H and Rempe G 2004 Nature 42850

[33] Fortier K M, Kim S Y, Gibbons M J, Ahmadi P and Chapman M S 2007 Phys. Rev. Lett. 98233601

[34] Katori H, Takamoto M, Pal'chikov V G and Ovsiannikov V D 2003 Phys. Rev. Lett. 91173005

[35] Brusch A, LeTargat R, Baillard X, Fouche M and Lemonde P 2006 Phys. Rev. Lett. 96103003

[36] Barber Z W, Hoyt C W, Oates C W, Hollberg L, Taichenachev A V and Yudin V I 2006 Phys. Rev. Lett. 96083002

[37] Maunz P, Puppe T, Schuster I, Syassen N, Pinkse P W H and Rempe G 2005 Phys. Rev. Lett. 94033002 